\title{
Exame Neuropsicológico no Diagnóstico Diferencial das Demências Primárias
}

\author{
Ivan Hideyo Okamoto* \\ Paulo Henrique Ferreira Bertolucci**
}

\section{RESUMO}

As doenças degenerativas primárias abrangem a maioria das causas de demência, com a avaliação neuropsicológica contribuindo com o diagnóstico diferencial e auxiliando na avaliação da gravidade do comprometimento cognitivo. A doença de Alzheimer é a principal causa de demência primária, com comprometimento em testes de avaliação de memória explícita (declarativa, episódica), destacando-se das demais áreas cognitivas. Outras causas de demência, como demência por corpúsculos de Lewy e demências fronto-temporais apresentam maior alteração em testes de função executiva. A avaliação neuropsicológica na doença de Huntington revela um comprometimento precoce na memória explícita (dificuldade no processamento de informações). O exame neuropsicológico nas demais causas de demências não possui particularidades que as diferenciem.

\section{UNITERMOS}

Demências primárias, diagnóstico, testes neuropsicológicos.
Pós-graduando da Disciplina de Neurologia da Escola Paulista de Medicina - UNIFESP.

** Professor Adjunto, Chefe do Setor de Neurologia do Comportamento da Escola Paulista de Medicina - UNIFESP.
Demência é uma síndrome de comprometimento cognitivo e comportamental, severo o suficiente para interferir com a atividade de vida diária e a qualidade de vida. Existem cerca de 70 doenças que podem causar demência, entretanto nem todas são progressivas. As demências ocorrem primariamente em fases mais tardias da vida, com uma prevalência de $1 \%$ aos 60 anos, dobrando a cada 5 anos até atingir 30\% a $50 \%$ aos $85 \operatorname{anos}^{1}$. O diagnóstico preciso das síndromes demenciais é importante para detectar causas reversíveis de demência, permitindo ao médico predizer o curso da doença, facilitando o planejamento do paciente e da família quanto a suas atividades sociais, além disso, a padronização do enfoque diagnóstico nas demências é importante na pesquisa clínica, incluindo estudos epidemiológicos e terapêuticos.

Embora existam diversas causas de demência, a doença da Alzheimer (DA) responde por cerca de $70 \%$ (isolada ou em associação) dos casos em países industrializados ${ }^{2}$. Em uma pequena porcentagem dos casos, uma etiologia reversível ou tratável poderá ser identificada como causa de demência, sendo os exemplos mais importantes as doenças da tireóide, neurossífilis, infecções por fungos, deficiências de vitaminas, e lesões estruturais do cérebro como tumores, hematomas subdurais e hidrocefalia (tabela 1).

As doenças degenerativas primárias abrangem a maioria das causas de demência (tabela 2), havendo critério clínico bem-estabelecido para alguns tipos de demência, como DA, demência com corpúsculos de Lewy e demência do tipo lobo frontal.

O diagnóstico clínico das demências inclui o teste neuropsicológico do paciente examinado, podendo ajudar no diagnóstico diferencial das diversas causas de demência, bem como avaliar o grau de severidade do comprometimento cognitivo. A exatidão do diagnóstico clínico (neuropsicológico), quando comparado com o diagnóstico anatomopatológico, varia de doença para doença, atingindo $91,4 \%$ na $\mathrm{DA}^{5}$ e $85 \%$ na demência vascular ${ }^{6}$.

Neste artigo tentaremos apresentar algumas características neuropsicológicas das diferentes causas de demência degenerativa primária que podem 


\section{TABELA 1}

Causas freqüentes de síndromes demenciais (CoreyBloom, 1996) ${ }^{3}$

- Doença de Alzheimer (DA)

- Demência associada com corpúsculos de Lewy

- DA e demência vascular (demência mista)

- Depressão

- Demência vascular

- Distúrbios metabólicos

- Intoxicação por drogas

- Infecções

- Lesões estruturais

- Demência secundária ao álcool

- Hidrocefalia

- Doença de Parkinson

- Demência de Pick e outras demências frontais

nos ajudar no diagnóstico diferencial entre elas, bem como, no caso de DA, mostrar algumas características que se diferenciam no estadiamento da doença.

O exame neuropsicológico, em geral, inicia-se com um teste breve, abrangente e simples, que funciona como triagem para detecção do comprometimento cognitivo, sendo os mais utilizados, o miniexame do estado mental ${ }^{7}$ e a escala de demência de Blessed ${ }^{8}$. Esses testes devem ser aplicados de maneira criteriosa, adaptados para idade e escolaridade ${ }^{9}$, não sendo aplicáveis como instrumento único para diagnóstico e acompanhamento das demências.

\section{DOENÇA DE ALZHEIMER}

A principal característica cognitiva em DA é o comprometimento progressivo da memória, entretanto os testes dessa área cognitiva são aplicáveis apenas nos estágios mais precoces da doença (leve e moderado), pois os pacientes em estágio severo não são passíveis de teste.

Memória de trabalho (working memory, memória primária): tanto para informações verbais como para visuoespaciais, parece haver comprometimento na DA leve, embora haja controvérsias. Tipicamente essa memória é testada por meio do span de dígitos, que
TABELA 2

Doenças degenerativas do SNC causando demência (Mayeux, Foster, Rossor e Whitehouse, 1993) ${ }^{4}$

- Doença de Alzheimer (DA)

- Doença de Pick

- Doença de Huntington

- Doença de Parkinson

- Paralisia supranuclear progressiva

- Síndrome de Hallervorden-Spatz

- Epilepsia mioclônica progressiva

- Gliose subcortical progressiva

- Complexo demência-parkinsonismo - ELA

- Degeneração do lobo frontal do tipo não Alzheimer

consiste no indivíduo repetir seqüências de números que acabou de ouvir; podem ainda ser solicitados a reproduzir seqüências de "toque de blocos" como no Corsi Block Tapping Test ${ }^{10}$.

Memória explícita (declarativa, episódica): os pacientes devem ter comprometimento desse subsistema de memória para preencher os critérios diagnósticos do DSM-IV ${ }^{11}$ de demência. O déficit de aprender informações, no nível episódico, aparece precocemente e está sempre presente, ou seja, o aprendizado de eventos e pessoas, que é importante têmporo-espacialmente para o conhecimento autobiográfico, está prejudicado. Outra marca da DA é a dificuldade em resolver problemas do dia-a-dia e de planejar atividades corretamente (secundárias ao déficit de aprendizado de informações). Um déficit em evocar fatos e eventos, principalmente os adquiridos mais recentemente, também está presente, é proporcional ao déficit de aprendizado episódico e pode ser percebido na dificuldade dos pacientes em reconhecer locais e a relação de pessoas e objetos com esses locais. Isso explica a confusão, precocemente notada nos indivíduos, quando têm de enfrentar mudanças rápidas de cena e locais ${ }^{12}$. Testes aqui utilizados são vários, como teste da lista de palavras, teste de aprendizado de associação pareada, evocação de história (memória lógica), entre outros.

Memória implícita (não-declarativa, semântica, procedural): podemos testar de diferentes maneiras esse subsistema, havendo muita discussão a respeito do 
comprometimento ou não de suas diversas áreas. Eslinger e Damasio ${ }^{13}$ demonstraram que o aprendizado de habilidades, por testes sensório-motores como o teste de perseguição rotatória (outros testes dessa área são o desenho pelo espelho e o teste de leitura invertida), está preservado em pacientes com DA, apesar do desempenho inicial desses indivíduos estar abaixo daquele da população-controle. Heindel et al. ${ }^{14}$ também encontraram preservação no aprendizado desse teste, entretanto encontraram comprometimento no teste de pré-ativação verbal (priming). Alguns testes de avaliação de priming verbal (priming de complementação de palavras) consistem em ler palavras (por exemplo: cadeira), sendo posteriormente apresentadas as letras iniciais (por exemplo: C-A-D-) e solicitado para completar com a primeira palavra que vier à cabeça. Esses testes apresentam discussão quanto aos resultados encontrados, com alguns estudos demonstrando uma redução significativa no teste de priming na complementação de palavras ${ }^{14,15,16}$ nos pacientes, enquanto outros estudos não apresentam comprometimento nos testes de memória procedural ${ }^{17}$. Então, pacientes com DA têm evidências de bases neurais separadas para o aprendizado sensório-motores de habilidades, preservado em muitos demenciados leves; para a pré-ativação verbal comprometida em outros tantos pacientes. Diferente do que ocorre, por exemplo, na doença de Huntington (doença degenerativa, que inclui demência como critério diagnóstico), em que ocorre comprometimento nos testes de aprendizado de habilidades, e preservação no desempenho de testes de pré-ativação verbal ${ }^{18}$.

Linguagem: os déficits de linguagem observados precocemente na DA podem ser notados com a dificuldade em nomear objetos (teste de nomeação de Boston), análise de discurso, vocabulário, capacidade descritiva e compreensão de leitura. A fala pode tornar-se um pouco lenta, podendo haver perseveração, repetição de palavras e frases fora de contexto. O teste de fluência verbal é muito sensível e utilizado para avaliar linguagem em estágios precoces da DA. Consiste em solicitar ao paciente, dentro de um tempo determinado, a falar o maior número de palavras possíveis que comecem com certa letra (categoria simbólica) ou de determinada categoria semântica (por exemplo: animais). Na DA leve, a categoria simbólica parece estar mais comprometida que a categoria semântica, embora em DA moderada e severa, as duas categorias estejam igualmente comprometidas ${ }^{19}$. Em estágios iniciais da DA, as capacidades léxicas e semânticas parecem ser mais vulneráveis à doença, enquanto as capacidades sintáticas e fonológicas estão relativamente preservadas $^{20}$. Nos estágios moderados da doença, as alterações incluem parafasias (paragramáticas), permanecendo morfemas sintáticos, apesar de haver algumas trocas semânticas e neologismos. Desenvolvem ainda dificuldade de compreensão com a evolução da doença (afasia de Wernicke ou afasia transcortical). Na fase severa da DA, há uma diminuição da fluência, ecolalia, perseveração e sons ininteligíveis, como grunhido e murmúrio, estando a capacidade de comunicação global severamente comprometida ${ }^{21}$.

Atenção: déficits de atenção seletiva, medidos por testes de tempo de reação, com lentificação do tempo, foram demonstradas na $\mathrm{DA}^{22}$. Outros testes utilizados, e que também apresentaram alterações, são o teste de símbolo de dígitos, teste das trilhas e teste de Stroop ${ }^{10}$, embora existam relatos de desempenho normal nos testes de atenção dos pacientes com $\mathrm{DA}^{23}$.

Demais áreas cognitivas: as funções visuoespaciais estão comprometidas no curso da doença, com os pacientes perdendo-se, com desorientação espacial, e dificuldade em manusear aparelhos complexos. Os testes visuoespaciais podem ser testes de desenho, cópia, orientação de linhas, construção com blocos, labirinto, leitura de mapa, entre outros ${ }^{21}$. As funções executivas podem estar comprometidas, porém parece não ocorrer em estágios iniciais da doença ${ }^{17}$.

\section{DEMÊNCIA COM CORPÚSCULOS DE LEWY}

O diagnóstico clínico de demência com corpúsculos de Lewy (DL) inclui um progressivo declínio cognitivo, que interfere nas atividades de vida diária do indivíduo. Além das alterações cognitivas, que discutiremos a seguir, deve apresentar outros sinais e sintomas como alucinações visuais recorrentes (em geral bem estruturadas e detalhadas) e alterações motoras de parkinsonismo. Pode apresentar história de quedas, síncopes, perdas transitórias de consciência, hipersensibilidade a neurolépticos. Uma das características em DL é a flutuação das funções cognitivas, havendo a possibilidade de o indivíduo alternar períodos (horas ou semanas) de profundo déficit cognitivo com períodos de quase-normalidade ${ }^{24}$. Quando comparados com o exame anatomopatológico, o diagnóstico clínico pode atingir até $90 \%$ de sensibilidade e $97 \%$ de especificidade no diagnóstico de $\mathrm{DL}^{25}$.

Testes de exame mental podem confirmar a presença 
de alteração cognitiva, mas podem ser insuficientes para diferenciar DL de DA e outras demências, necessitando de teste neuropsicológico mais detalhado.

Memória: sintomas de alteração da memória persistente nem sempre estão presentes no início da doença, porém tornam-se evidentes com a evolução da doença. Os pacientes com DL estão particularmente comprometidos nos testes de evocação da memória, enquanto na DA parece haver prejuízo na aquisição e consolidação da memória.

Demais áreas cognitivas: os indicadores clínicos no diagnóstico de DL são o mal desempenho nos testes de funções executivas e resolução de problemas, como nos testes de Wisconsin (Wisconsin card sorting Test), teste das trilhas (Trail Making), e fluência verbal (por letras e categorias), com relativa preservação das funções nos testes de desempenho visuoespacial (construção com blocos, desenho do relógio ou cópia de figuras). Com o progredir da demência, essas diferenças podem perderse, tornando difícil o exame clínico e a diferenciação com outras demências em estágio severo ${ }^{24}$.

\section{DEMÊNCIAS FRONTO-TEMPORAIS (INCLUINDO DOENÇA DE PICK)}

As manifestações precoces das demências frontotemporais (DFT), apesar de representar um grupo heterogêneo de doenças com características clínicas e patológicas diversas, são relacionadas às alterações de comportamento; podendo apresentar-se como isolamento social, depressão, psicose, perda de crítica, desinibição e irritabilidade. Essas alterações podem preceder em alguns anos as alterações intelectuais, mas representam as características diferenciais desse tipo de demência.

Os testes neuropsicológicos de triagem, como o MEEM, podem estar normais no início da doença ${ }^{26}$, porém variam com a severidade da demência.

Memória: os pacientes com DFT são tipicamente bem orientados no tempo e espaço, sendo capazes de fornecer informações; entretanto, desempenham-se mal nos testes formais de memória. Essa dissociação pode representar uma falha de estratégia na utilização da memória, mais até que uma dificuldade em adquirir e reter informações ${ }^{27}$.

Linguagem: segundo os critérios clínicos e neuropatológicos das $\mathrm{DFT}^{28}$, os distúrbios de linguagem caracterizam-se pela progressiva redução da fala (falta de espontaneidade e economia na forma de falar), estereotipia da fala (repetição de repertório limitado de palavras, frases e temas), ecolalia e perseveração, e mais tardiamente, mutismo.

Demais áreas cognitivas: outras características que diferenciam DFT são o déficit de iniciação, atingir objetivos e planejamento (funções executivas), em fases mais tardias da doença. Outras alterações como apraxia e funções visuoespaciais não são comprometidas com freqüência ${ }^{29}$.

\section{DOENÇA DE HUNTINGTON}

A doença de Huntington (DH) é uma doença neurodegenerativa autossômica dominante, caracterizada por movimentos anormais, demência e alterações psiquiátricas. O quadro demencial tem características psiquiátricas predominantes, incluindo delírios, depressão e mania. As alterações cognitivas colocam a DH em um grupo de demências também chamadas de "demências subcorticais", junto com a doença de Parkinson, a paralisia supranuclear progressiva e a doença de Wilson; aqui, sintomas como afasia, apraxia e agnosia, marcadamente de envolvimento cortical, não são vistos com freqüência ${ }^{30}$.

Memória: está comprometida e aparece precocemente na evolução da doença, apresentando dificuldade em se lembrar de fatos e eventos públicos, assim como em testes de evocação (memória explícita); entretanto, parece haver mais um déficit em processar informações e uma falha na estratégia de evocação do que aquisição e consolidação da memória como ocorre na DA. Nos testes de aprendizado motor e de pré-ativação léxica (priming), que avaliam memória implícita, os indivíduos com DH têm desempenho normal no teste de priming, e prejuízo no teste de perseguição motora (oposto ao que acontece em DA) ${ }^{14}$.

Demais áreas cognitivas: a linguagem não tem comprometimento tão importante como nas demências corticais, mas parece haver discussão entre os autores ${ }^{31}$, com os trabalhos demonstrando comprometimento em alguns testes que precisam de um acesso eficiente a reserva léxica (WAIS-R vocabulary subtest, teste de nomeação de Boston e fluência verbal). O comprometimento de funções visuoespaciais é marcante em $\mathrm{DH}$, como na cópia da figura complexa de Rey-Osterrieth e teste de orientação espacial (Standardized road map test of directional sense), relacionando-os a uma percepção anormal do espaço pessoal ${ }^{30}$. 


\section{DOENÇA DE PARKINSON}

A doença de Parkinson (DP) é a causa mais comum de demência associada com alterações extrapiramidais. Em um estudo, mais de $65 \%$ dos pacientes com DP desenvolveram demência aos $85 \operatorname{anos}^{31}$. Na DP com demência, também ocorre alteração de memória, dificuldade em manter atenção, porém as alterações cognitivas corticais não são muito evidentes, como afasia e apraxia, pelo menos em estágios iniciais da doença.

Memória: em geral os trabalhos sugerem um comprometimento de memória com capacidade preservada em registrar, armazenar e consolidar informações, porém com dificuldade em utilizar adequadamente essas informações. Os testes para avaliar a memória primária (working memory) parecem estar comprometidos devido ao déficit de atenção dos pacientes com DP, ou dependem dos recursos de atenção solicitados em cada teste ${ }^{32}$. A memória explícita (declarativa) está preservada para testes de reconhecimento com estímulo verbal e visuoespacial ${ }^{33,34}$, porém podem estar comprometidos quando os testes implicam organizar a informação mentalmente, escolher e organizar de forma ativa a resposta, principalmente quando o material a ser aprendido não está semanticamente organizado (lista de palavras, Rey Auditory Verbal Learning Test, teste de evocação seletiva de Buschke) ${ }^{32}$.

A memória implícita, abrangendo aprendizado procedural e priming léxico, parece estar comprometida nos pacientes demenciados com DP, porém estaria preservada nos não-demenciados com $\mathrm{DP}^{14}$.

Linguagem: são freqüentes as alterações de fala, tornando-se monótona, com diminuição do uso de parâmetros vocais para demonstrar ênfase e alteração. A articulação é imprecisa, por vezes caracterizando uma disartria hipocinética. Outro fenômeno que ocorre é a aceleração do ritmo de fala (taquifemia), e menos freqüentemente a repetição compulsiva de palavras e frases (palilalia). Há ainda uma diminuição na velocidade do movimento articulatório, e possivelmente uma alteração no planejamento da fala, assim como ocorre em outras partes do corpo; entretanto, a taxa de deterioração da fala nem sempre acompanha as alterações motoras (bradicinesia) ${ }^{35}$.

Demais áreas cognitivas: há comprometimento no desempenho de testes visuoespaciais, quer por alteração visuomotora, quer por alteração visuoperceptual, podendo estar relacionadas a um comprometimento de representação interna. Testes de função executiva (Wisconsin card sorting test, teste das trilhas $\mathrm{B}$, teste de Stroop, fluência verbal, torre de Hanói e variantes) estão prejudicados nos pacientes com $\mathrm{DP}^{32}$.

\section{PARALISIA SUPRANUCLEAR PROGRESSIVA (SÍNDROME DE STEELE-RICHARDSON-OLSZEWSKI)}

A paralisia supranuclear progressiva (PSP) é uma doença progressiva, crônica, caracterizada por rigidez extrapiramidal, distonia axial, labilidade pseudobulbar de afeto, dificuldade de deglutição, dificuldade severa de marcha, oftalmoplegia supranuclear e demência. O quadro demencial não é um critério necessário para o diagnóstico, entretanto ocorre em $60 \%$ a $80 \%$ dos pacientes com PSP.

O comprometimento cognitivo da PSP pode ser atribuído a alterações subcorticais, como esquecimento, lentificação do pensamento, mudanças de personalidade mais do que afasia, agnosia ou alterações visuoespaciais, e as alterações são similares, porém mais severas que na $\mathrm{DP}^{36}$. Particularmente, os indivíduos com PSP apresentam alterações em testes que solicitam movimentos seqüenciais e mudança de conceito, e outros testes de função de lobo frontal. Além desses, testes de procura visual, fluência verbal, span de dígitos, memória verbal e memória lógica podem estar comprometidos na PSP ${ }^{37}$.

\section{GLIOSE SUBCORTICAL PROGRESSIVA}

A gliose subcortical progressiva (GSP) é uma doença neurodegenerativa rara, freqüentemente familiar, com início na quarta até sexta décadas. O curso é progressivo por 5 a 30 anos. Inicialmente, é caracterizada por mudanças emocionais, de personalidade e psiquiátricas, com delírios, paranóia, alucinações auditivas, depressão, idéias suicidas, perda de julgamento e comportamento social. Mais tarde, as mudanças cognitivas tornam-se evidentes, afetando memória, raciocínio e percepção visual, não podendo ser distinguidas clinicamente da doença de Pick. Achados neurológicos focais não fazem parte da síndrome, porém mutismo pode ocorrer em fases tardias da doença. O exame microscópico mostra intensa astrogliose da substância branca subcortical, principalmente na junção córtico-subcortical ${ }^{37}$.

\section{COMPLEXO ESCLEROSE LATERAL AMIOTRÓFICA - DEMÊNCIA}

Esta doença tem sido descrita no Japão e em países do Oriente, como um quadro de demência leve a moderada associado com amiotrofia. Na maioria dos casos, as mudanças de personalidade iniciam o quadro ${ }^{38}$. A demência é descrita com comprometimento de funções 
fronto-temporais, por vezes não sendo possível distinguir das alterações cognitivas encontradas na doença de Pick ${ }^{39}$.

\section{EPILEPSIA MIOCLÔNICA PROGRESSIVA}

A epilepsia mioclônica progressiva (EMP) abrange um grupo de doenças caracterizadas por mioclonias espontâneas e de ação, crises generalizadas, por vezes ataxia, e variáveis níveis de comprometimento intelectual ${ }^{40}$. Alguns autores colocam a DA de início precoce como causa de $\mathrm{EMP}^{41}$.

\section{SUMMARY}

Neuropsychological examination in the differential diagnosis of primary dementias

Primary degeneration of the Central Nervous System is the main cause of dementia. Neuropsychological examination may be helpful not only in stablishing the etiology, but also the level of cognitive deficits. Most of the so called primary dementia cases are due to Alzheimer's disease, in wich the first disturbances are in explicit episodic memory, at a time when other functions are largely spared. Other causes of dementia, like fronto-temporal dementia and Lewy body disease show disturbance in executive functions. In Huntington's disease there is an early disturbance of explicit memory with slowing of information processing. Though some patterns can be identified (eg slowing of processing and difficulty in shifting strategies in subcortical dementias), other causes of dementia do not have specific changes at the neuropsychological examination.

\section{KEY WORDS}

Primary dementias, diagnosis, neuropsychological examination.

\section{Referências}

1. Evans D.A.; Funkenstein H.H; Albert M.S; et al. Prevalence of Alzheimer's disease in a community population of older persons: higher than previously reported. JAMA, 262:25512556, 1989.

2. Kokmen E.; Beard C.M.; Offord K.P. and Kurland L.T. Prevalence of medically diagnosed dementia in a defined US population: Rochester, Minnesota. Neurology, 39:773-776, 1989.

3. Corey-Bloom J. The diagnosis and evaluation of dementia. In Dementia Update. American Academy of Neurology $-48^{\text {th }}$ Annual Meeting - San Francisco 441:1-19,1996.

4. Mayeux R.; Foster N.L.; Rossor M.; Whitehouse P.J. The clinical evaluation of patients with dementia. In: Dementia. Whitehouse PJ, ed. Contemporary neurology series. Philadelphia: F.A. Davis, 4:92-129, 1993.

5. Becker J.T.; Boller F.; Lopez O.L.; Saxton J.; McGonigle K.L. The natural history of Alzheimer's disease. Arch Neurol, 51:585-594, 1994.

6. Erkinjuntti T.; Haltiam, Palo J.; et al. Accuracity of the clinical diagnosis of vascular dementia: a prospective clinical and post-mortem neuropatological study. J Neurol Neurosurg Psychiatry, 51:1037-1044, 1988.

7. Folstein M.F.; Folstein S.E.; McHugh P.R. "Mini- Mental State": a practical method of granding the cognitive state of patients for the clinician. J Psychiatric Res, 12:189-198, 1975.
8. Blessed G.; Tomlinson B.E. and Roth M. The association between quantitative measures of dementia and ofsenile change in the grey matter of elderly subjects. Br J Psychiatry, 114:797-811, 1968.

9. Brucki, S.M.D. Avaliação do desempenho no Mini-Exame do Estado Mental em pacientes de um hospital geral. São Paulo, 1993, Tese de Mestrado, Escola Paulista de Medicina.

10. Almkivist O. Neuropsychological features of early Alzheimer Disease preclinical and clinical stages. Acta Neurol Scand, 165(suppl.):63-71, 1996.

11. American Psychiatric Association. Diagnostic and Statistical manual of Mental Disorders, $4^{\text {th }}$ ed. (DSM-IV). Washington: American Psychiatric Association, 1994.

12. Damasio A.R.; Tranel D.; Damasio H. Amnesia caused by herpes simplex encephalitis, infarctions in basal forebrain, Alzheimer's disease and anoxia/ischemia. In: Handbook of Neuropsychology, Vol. 3. C F. Boller e J. Grafman (eds.). Elsevier Science publisers B.V. Amsterdam, 6:149-166, 1991.

13. Eslinger P.J.; Damasio A.R. Preserved motor learning in Alzheimer's disease: implications for anatomy and behavior. J Neurosci, 6:3006-3009, 1986.

14. Heindel WC, Salmon DP; Schults C.W.; et al. Neuropsychological evidence for multiple implicity memory systems: a comparison of Alzheimer's, Huntington's, an Parkinson's disease patients. J Neurosci, 9:582-587, 1989.

15. Shimamura A.P.; Salmon D.P.; Squire L.R.; Butters N. Memory dysfunction and word priming in dementia and amnesia. Behav Neurosci, 101:347-351, 1987.

16. Salmon D P. Shimamura Ap, Butters N. Lexical and semantic deficits in patients with A.D. J Clin Exp Neuropsychol, 10:477494, 1988

17. Bondi M.W.; Kasniak A.W. Implicit and explicit memory in AD and PD. J Clin Exp Neuropsychol, 13:339-358, 1991.

18. Heindel W.C.; Butters N.; Salmon D.P. Impaired learning of a motor skill in patients with Huntington's disease. Behav Neurosci, 102:141-147, 1988.

19. Rosen W.G. Verbal fluency in aging and dementia. J Clin Neuropsychol, 2:135-140, 1980.

20. Huff F.J. Language in normal aging and age - related neurological diseases. In: Handbook of neuropsychology, vol. 4 C. F. Boller e J. Grafman (eds.). Elsevier Science publisers B.V. Amsterdam, 12:251-264, 1990.

21. Kertesz A.; Mohs C. Cognition. In: Clinical diagnoses and management of Alzheimer's disease. S. Gautier (ed.). Martin Dunitz. London, 10:155-174, 1996.

22. Cronin-Golomb A., Corkin S., Rosen T.J. Neuropsychological assessment of dementia In: Dementia. Whitehouse PJ, ed. Contemporary neurology series. Philadelphia: FA Davis, 5:130-164, 1993.

23. Lines C.R.; Dawson C.; Preston G.C.; et al. Memory and attention in patients with senile dementia of $A D$ type and in normal elderly subjects. J Clin Neuropsychol, 13:691-702, 1991.

24. Mackeith I.G.; Galasko D.; Kosaka K.; et al. Consensus guidelines for the clinical and pathologic diagnosis of dementia with Lewy bodies (DLB): report of the consortium on DLB international workshop. Neurology, 47:1113-1124, 1996.

25. Mackeith I.G.; Fairbairn A.F.; Bothwell R.A.; et al. An evaluation of the predictive validity and inter-rater reliability of clinical diagnostic criteria for senile dementia of Lewy body type. Neurology, 44:872-877, 1994.

26. Geldmacher D.S.; Whitehouse Jr. P.J. Diferential diagnosis of Alzheimer's disease. Neurology, 48(suppl.): S2-S9, 1997.

27. Neary D.; Snowden J.S.; Northen B.; Goulding P. Dementia of frontal lobe type. J Neurol Neurosurg Psychiatry, 51: 353$361,1988$. 
28. Lund and Manchester groups. Clinical and neuropathological criteria for frontotemporal dementia. J Neurol Neurosurg Psychiatriy, 57: 416-418, 1994.

29. Geldmacher D.S.; Whitehouse Jr. P.J. Evaluation of dementia New Eng $J$ of Medicine, 335: 330-336, 1996.

30. Brandt J. Cognitive impairments in Huntington's disease: insights into the neuropsychology of the striatum. In: Handbook of Neuropsychology, Vol. 5. C. F. Boller e J. Grafman (eds.). Elsevier Science publisers B.V. Amsterdam. 1991. 11: 241-264.

31. Mayeux R.; Chen J.; Mirabello E.; et al. An estimate of the incidence of dementia in Parkinson's disease. Neurology, 40:1513-1516, 1990.

32. Dubois B.; Boller F.; Pillon B. et al. Cognitive deficits in Parkinson's disease. In: Handbook of Neuropsychology, Vol 5. Boller F. e Grafman (eds.) Elsevier Science Publishers B.V. Amsterdam, 10:195-240, 1991.

33. El Awar M.; Becker J.T.; Hammond K.M.; et al. Learning deficits in Parkinson's disease: comparative with Alzheimer's disease and normal aging. Arch Neurol, 44:180-184, 1987.

34. Taylor A.E.; Saint-Cyr J.A.; Lang A.E. Frontal lobe dysfunction in Parkinson's disease. Brain, 109:845-883, 1986.

35. Critchley EMR. Speech disorders of parkinsonism: a review. J Neurol Neurosurg Psychiatry, 44:751-758, 1981.

36. Maher E.R.; Lees A.J. The clinical features and natural history of the Steele-Richardson-Olszewski syndrome. Neurology, 36:1005-1008, 1986.
37. Civil R.H.; Whitehouse P.J.; Lanska D.J.; et al. Degenerative dementias. In: Dementia. Whitehouse PJ, ed. Contemporary neurology series. Philadelphia: FA Davis, 6:167-214, 1993.

38. Morita K.; Halya H.; Ikeda T.; Namba M. Presenile dementia combined with amyotrophy: A review of 34 Japanese cases. Arc Gerontol Geriatrics, 6:263-277, 1987.

39. Montgomery G.K.; Erickison L.M. Neuropsychological perspectives in amyotrofic lateral sclerosis. Neurol Clin, 5:6181, 1987.

40. Marseille Consensus Group. Classification of progressive myoclonus epilepsies and related disorders. Ann Neurol, 28:113-116, 1990.

41. Melanson M.; Nalbantoglu J.; Berkovic S.; et al. Progressive myoclonus epilepsy in young adults with neuropathologic features of Alzheimer's disease. Neurology, 49:1732-1733, 1997.

\section{Endereço para correspondência:}

Ivan Hideyo Okamoto

Universidade Federal de São Paulo

Escola Paulista de Medicina - Disciplina de Neurologia

Setor de Neurologia do Comportamento

Rua Botucatu, 740 - Vila Clementino

CEP 04023-900 São Paulo (SP)

E-mail: hideyo@sti.com.br 